\title{
Modified exponential function method for the KP-BBM equation
}

\author{
Tolga Aktürk ${ }^{a, *}$, Gülnur Yel ${ }^{b}$ \\ ${ }^{a}$ Department of Mathematics and Science Education, Faculty of Education, Ordu University, Turkey. \\ ${ }^{b}$ Faculty of Educational Sciences, Final International University, Kyrenia, Mersin 10, Turkey.
}

\begin{abstract}
In this study, the travelling wave solutions of the Kadomtsev-Petviashvili-Benjamin-Bona- Mahony equation were obtained by using modified exponential function method. This method provides the solution of nonlinear partial differential equation by using exponential function. The submitted solutions are implied in terms of the hyperbolic functions, trigonometric functions. The 2D and 3D graphics and contour simulations of these solution functions were obtained by using computational program.
\end{abstract}

Keywords: The nonlinear equations, the Kadomtsev-Petviashvili-Benjamin-Bona-Mahony equation (KP-BBM), the modified exponential function method (MEFM).

2010 MSC: 35Q99, 35C07.

(C)2020 All rights reserved.

\section{Introduction}

Solutions of nonlinear partial differential equations have an important place in various fields. There are various methods in the literature to find solutions to such equations. Some of these are as follows; Kudryashov's method [30, 35], the sine-Gordon expansion method [9, 19], the new function methods $[1,2,13]$, the trial equation method $[31,34]$ the extended trial equation method $[39,40]$, the generalized Bernoulli sub-equation function method $[7,14,44]$ and so on $[3-6,10,11,15-18,20-25,27,29,33,36-$ $38,41,42,45-50]$. In this study, we apply the modified exponential function method (MEFM) [8, 9, 26] to solve the Kadomtsov-Petviashvili-Benjamin-Bona-Mahony (KP- BBM) equation [12, 28, 32, 43, 51].

$$
\left(u_{t}+u_{x}-a\left(u^{2}\right)_{x}-b u_{x x t}\right)_{x}+k u_{y y}=0 .
$$

\section{Analysis of the Method}

The summary of the modified exponential function method is given in the following steps [26].

Consider the general form of nonlinear partial differential equation;

$$
P\left(u, u_{x}, u_{y}, u_{t}, u_{x x}, u_{y y}, u_{t t}, \cdots\right)=0,
$$

where $u=u(x, y, t)$ is unknown function, $P$ is a polynomial that has $u(x, y, t)$ function and its partial

\footnotetext{
*Corresponding author

Email addresses: tolgaakturkk@gmail .com (Tolga Aktürk), gulnur . yel@final .edu.tr (Gülnur Yel)

doi: $10.22436 / \mathrm{mns} .06 .01 .01$
}

Received: 2019-05-06 Revised: 2019-10-29 Accepted: 2019-12-04 
derivatives respect to $x, y$ and $t$.

Step 1. Suppose that the travelling wave transformation as,

$$
u(x, y, t)=u(\zeta), \quad \zeta=x+y-c t
$$

where $\mathrm{c}$ is a nonzero constant that can be determined later. If the equation (2.1) is obtained by substituting the necessary derivatives Eq. (2.2), the following nonlinear ordinary differential equation is obtained.

$$
\mathrm{N}\left(\mathrm{U}, \mathrm{U}^{\prime}, \mathrm{U}^{\prime \prime}, \mathrm{u}^{\prime \prime \prime}, \cdots\right)=0 \text {. }
$$

Step 2: Let's consider the following solution function for the equation;

$$
u(\zeta)=\frac{\sum_{i=0}^{N} A_{i}[\exp (-\Omega(\zeta))]^{i}}{\sum_{j=0}^{M} B_{j}[\exp (-\Omega(\zeta))]^{j}}=\frac{A_{0}+A_{1} \exp (-\Omega)+\cdots+A_{N} \exp (N(-\Omega))}{B_{0}+B_{1} \exp (-\Omega)+\cdots+B_{M} \exp (M(-\Omega))^{\prime}}
$$

where $A_{i}, B_{j},(0 \leqslant i \leqslant N, 0 \leqslant j \leqslant M)$ are constants can be determined. $A_{N} \neq 0, B_{M} \neq 0$, and $\Omega=\Omega(\zeta)$ solves the following ordinary differential equation;

$$
\Omega^{\prime}(\zeta)=\exp (-\Omega(\zeta))+\mu \exp (\Omega(\zeta))+\lambda
$$

When we solve the Eq. (2.5), we get the solution families as follows [3, 41].

Family 1: When $\mu \neq 0, \lambda^{2}-4 \mu>0$,

$$
\Omega(\zeta)=\ln \left(\frac{-\sqrt{\lambda^{2}-4 \mu}}{2 \mu} \tanh \left(\frac{\sqrt{\lambda^{2}-4 \mu}}{2}(\zeta+E)\right)-\frac{\lambda}{2 \mu}\right) .
$$

Family 2: When $\mu \neq 0, \lambda^{2}-4 \mu<0$,

$$
\Omega(\zeta)=\ln \left(\frac{\sqrt{-\lambda^{2}+4 \mu}}{2 \mu} \tan \left(\frac{\sqrt{-\lambda^{2}+4 \mu}}{2}(\zeta+E)\right)-\frac{\lambda}{2 \mu}\right) .
$$

Family 3: When $\mu=0, \lambda \neq 0$, and $\lambda^{2}-4 \mu>0$,

$$
\Omega(\zeta)=-\ln \left(\frac{\lambda}{\exp (\lambda(\zeta+E))-1}\right)
$$

Family 4: When $\mu \neq 0, \lambda \neq 0$ and $\lambda^{2}-4 \mu=0$,

$$
\Omega(\zeta)=\ln \left(-\frac{2 \lambda(\zeta+E)+4}{\lambda^{2}(\zeta+E)}\right) .
$$

Family 5: When $\mu=0, \lambda=0$ and $\lambda^{2}-4 \mu=0$,

$$
\Omega(\zeta)=\ln (\zeta+E) .
$$

where $A_{0}, A_{1}, \ldots, A_{N}, B_{0}, B_{1}, \ldots, B_{M}, E, \lambda$, $\mu$ are constants. Using the balancing principle, the relationship between $N$ and $M$, which is the upper boundary of the summation symbol in the equation (2.4).

Step 3: Substituting Eq. (2.5) along with solution families into Eq. (2.4) we have a polynomial of $\exp (\Omega(\xi))$. Algebraic equation system consisting of coefficients is obtained. Then, the coefficients of this system are solved and the solution function is obtained by substituting the equation (2.1). 


\section{Application}

Let use Eq. (2.2) along with Eq. (2.4) into Eq. (1.1). In this case, we have nonlinear ordinary differential equation as follows;

$$
(1+\mathrm{k}-\mathrm{c}) \mathrm{U}-\mathrm{aU}^{2}+\mathrm{bcU^{ \prime \prime }}=0 .
$$

Using the balancing term $\mathrm{U}^{\prime \prime}$ and $\mathrm{U}^{2}$, we get a relationship the following,

$$
M+2=N .
$$

Different cases can be obtained for suitable values of $M$ and $N$. We have chosen $M=1$ and $\mathrm{N}=3$ values, then some travelling wave solutions appeared as following submitted.

\section{Case 1:}

$$
A_{0}=\frac{6 b c \mu B_{0}}{a}, A_{1}=\frac{6 b c\left(\lambda B_{0}+\mu B_{1}\right)}{a}, A_{2}=\frac{6 b c\left(B_{0}+\lambda B_{1}\right)}{a}, A_{3}=\frac{6 b c B_{1}}{a}, k=-1+c-b c \lambda^{2}+4 b c \mu .
$$

Family 1:

$$
u_{1,1}(x, y, t)=\left(\frac{6 b c \mu\left(-\lambda^{2}+4 \mu\right)}{a\left(\lambda \operatorname{Cosh}\left[\frac{1}{2}(E E+\xi) \sqrt{\lambda^{2}-4 \mu}\right]+\sqrt{\lambda^{2}-4 \mu} \operatorname{Sinh}\left[\frac{1}{2}(E E+\xi) \sqrt{\lambda^{2}-4 \mu}\right]\right)^{2}}\right),
$$
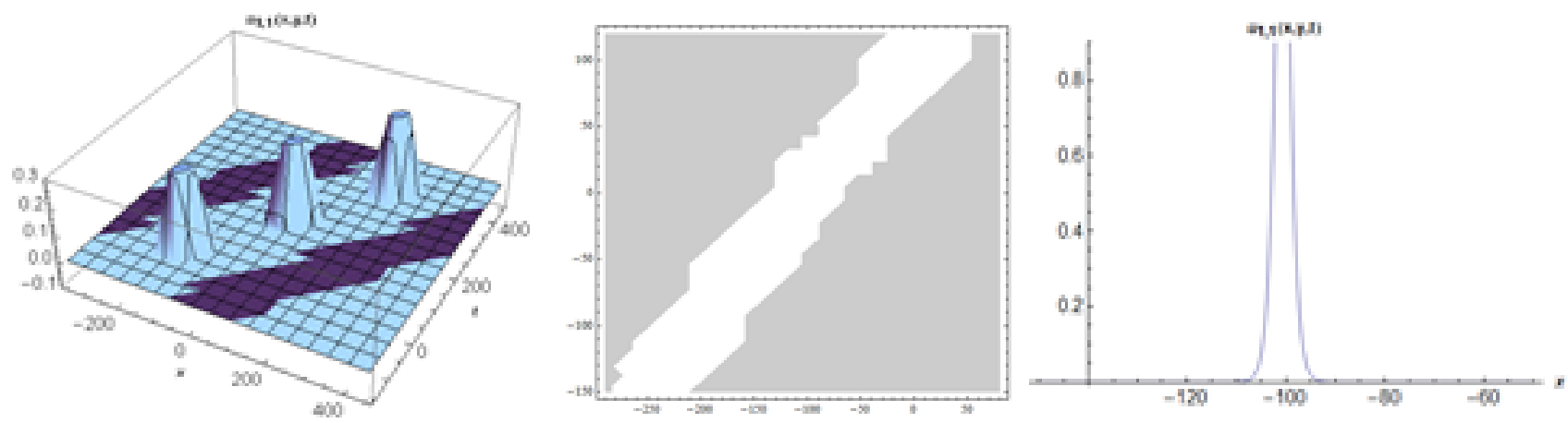

Figure 1: The 3D, contour graphic and $2 \mathrm{D}$ surfaces of Eq. (3.4) in $\lambda=3, \mu=2, c=1, a=-2, b=$ $2, \mathrm{EE}=100, \mathrm{t}=1$.

\section{Family 2:}

$$
u_{1,2}(x, y, t)=\left(\frac{6 b c \mu\left(-\lambda^{2}+4 \mu\right)}{a\left(\lambda \operatorname{Cos}\left[\frac{1}{2}(E E+\xi) \sqrt{-\lambda^{2}+4 \mu}\right]-\sqrt{-\lambda^{2}+4 \mu} \operatorname{Sin}\left[\frac{1}{2}(E E+\xi) \sqrt{-\lambda^{2}+4 \mu}\right]\right)^{2}}\right),
$$
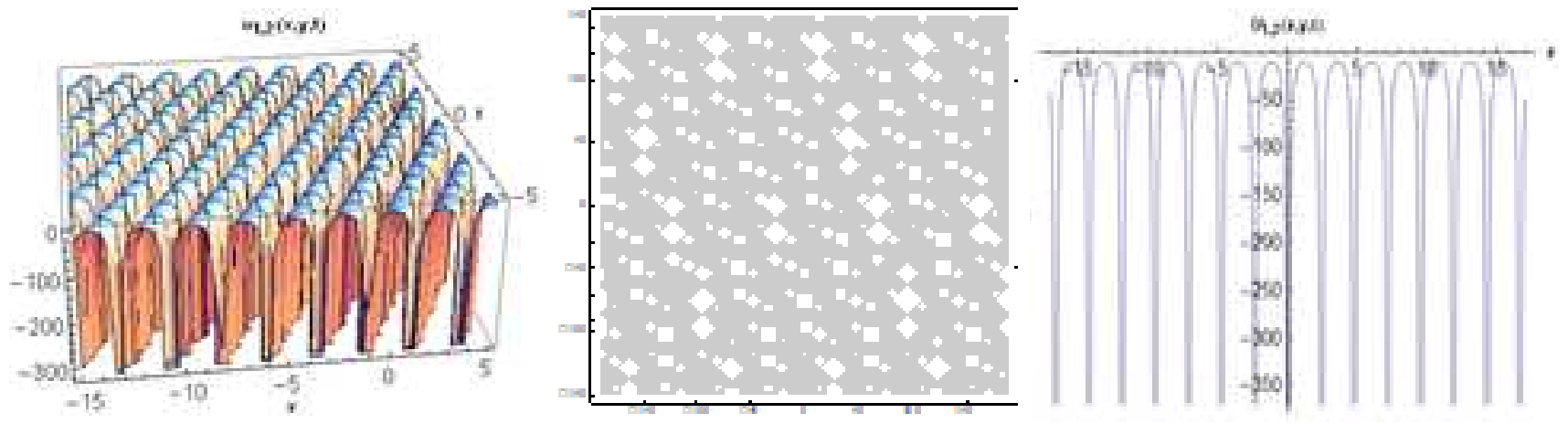

Figure 2: The 3D, contour graphic and $2 \mathrm{D}$ surfaces of Eq. (3.5) in $\lambda=1, \mu=2, c=1, a=-2, b=$ $2, \mathrm{EE}=100, \mathrm{t}=1$. 


\section{Case 2:}

$$
\begin{aligned}
& A_{0}=\frac{\left(\lambda^{2}+2 \mu\right) A_{3} B_{0}}{6 B_{1}}, A_{1}=\frac{1}{6} A_{3}\left(\lambda^{2}+2 \mu+\frac{6 \lambda B_{0}}{B_{1}}\right), A_{2}=A_{3}\left(\lambda+\frac{B_{0}}{B_{1}}\right), \\
& b=-\frac{a A_{3}}{a\left(\lambda^{2}-4 \mu\right) A_{3}-6(1+k) B_{1}}, c=1+k-\frac{a\left(\lambda^{2}-4 \mu\right) A_{3}}{6 B_{1}} .
\end{aligned}
$$

Family 1:

$$
u_{2,1}(x, y, t)=\frac{\left(\lambda^{2}-4 \mu\right) \operatorname{Sech}\left[\frac{1}{2} \psi\right]^{2}\left(-4 \mu+\left(\lambda^{2}-2 \mu\right) \operatorname{Cosh}[\psi]+\lambda \sqrt{\lambda^{2}-4 \mu} \operatorname{Sinh}[\psi]\right) A_{3}}{6 B_{1}\left(\lambda+\sqrt{\lambda^{2}-4 \mu} \operatorname{Tanh}\left[\frac{1}{2} \psi\right]\right)^{2}}
$$

where $\left(\psi=(E E+\xi) \sqrt{\lambda^{2}-4 \mu}\right)$.
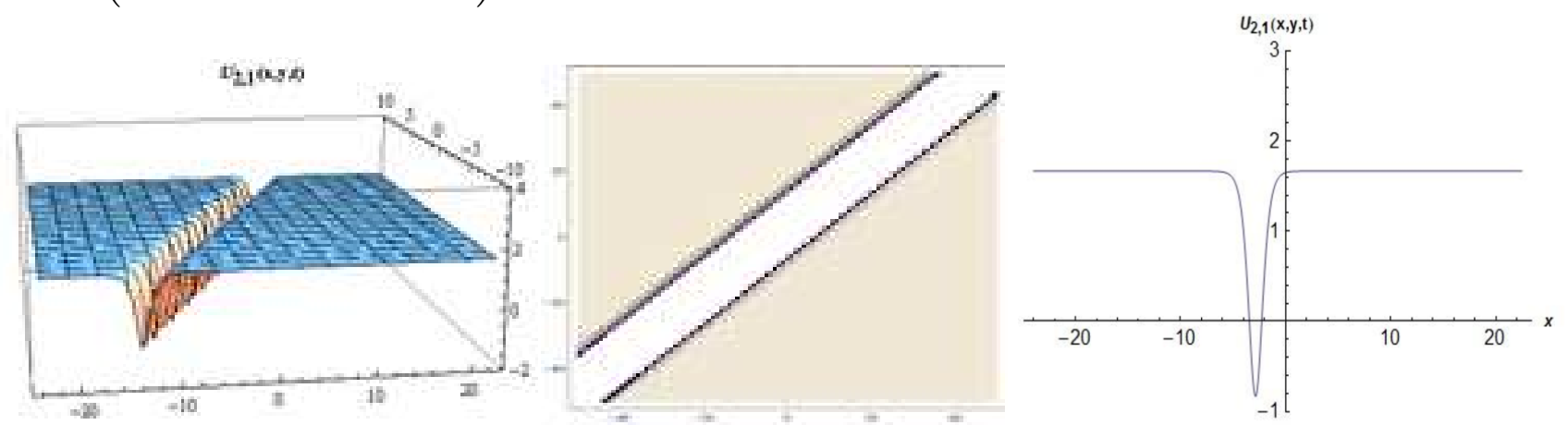

Figure 3: The 3D, contour graphic and $2 \mathrm{D}$ surfaces of Eq. (3.7) in $\lambda=3, \mu=1, \mathrm{c}=1, \mathrm{~A}_{3}=2, \mathrm{~B}_{1}=$ $1, \mathrm{EE}=1, \mathrm{t}=1$.

Family 2:

$$
u_{2,2}(x, y, t)=\frac{\left(\lambda^{2}-4 \mu\right) \operatorname{Sec}\left[\frac{1}{2} v\right]^{2}\left(-4 \mu+\left(\lambda^{2}-2 \mu\right) \operatorname{Cos}[v]-\lambda \sqrt{-\lambda^{2}+4 \mu} \operatorname{Sin}[v]\right) A_{3}}{6 B_{1}\left(\lambda-\sqrt{-\lambda^{2}+4 \mu} \operatorname{Tan}\left[\frac{1}{2} v\right]\right)^{2}}
$$

where $\left(v=(E E+\xi) \sqrt{-\lambda^{2}+4 \mu}\right)$.
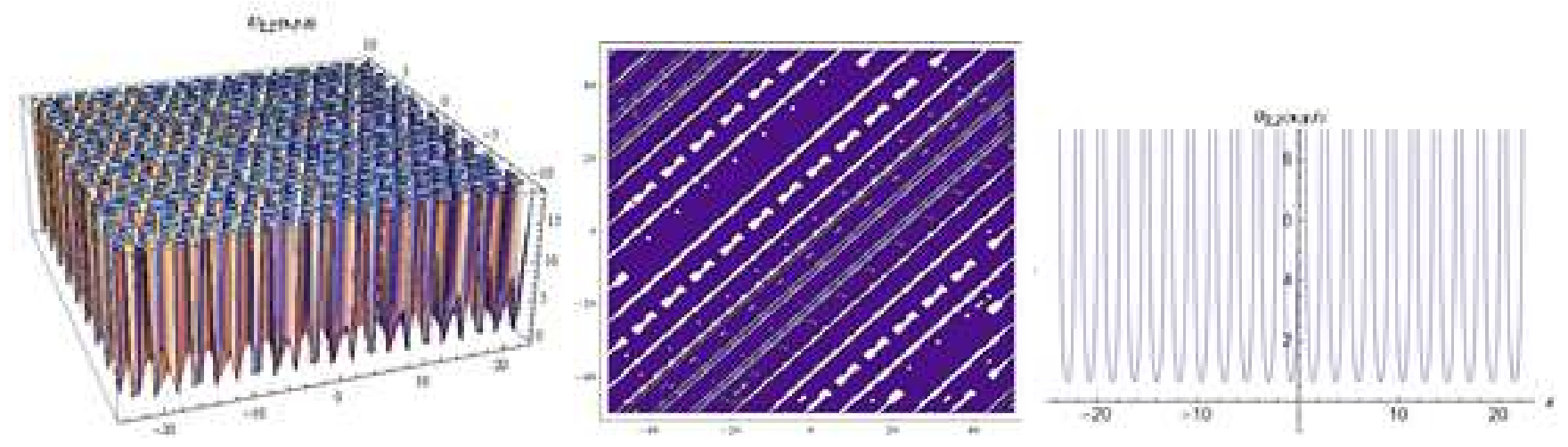

Figure 4: The $3 \mathrm{D}$, contour graphic and $2 \mathrm{D}$ surfaces of Eq. (3.8) in $\lambda=2, \mu=3, c=1, A_{3}=2, B_{1}=$ $2, \mathrm{EE}=100, \mathrm{t}=1$.

Family 4:

$$
u_{2,4}(x, y, t)=-\frac{\left(\lambda^{2}(-8+(E E+\xi) \lambda(4+(E E+\xi) \lambda))-4(2+(E E+\xi) \lambda)^{2} \mu\right) A_{3}}{12(2+(E E+\xi) \lambda)^{2} B_{1}},
$$



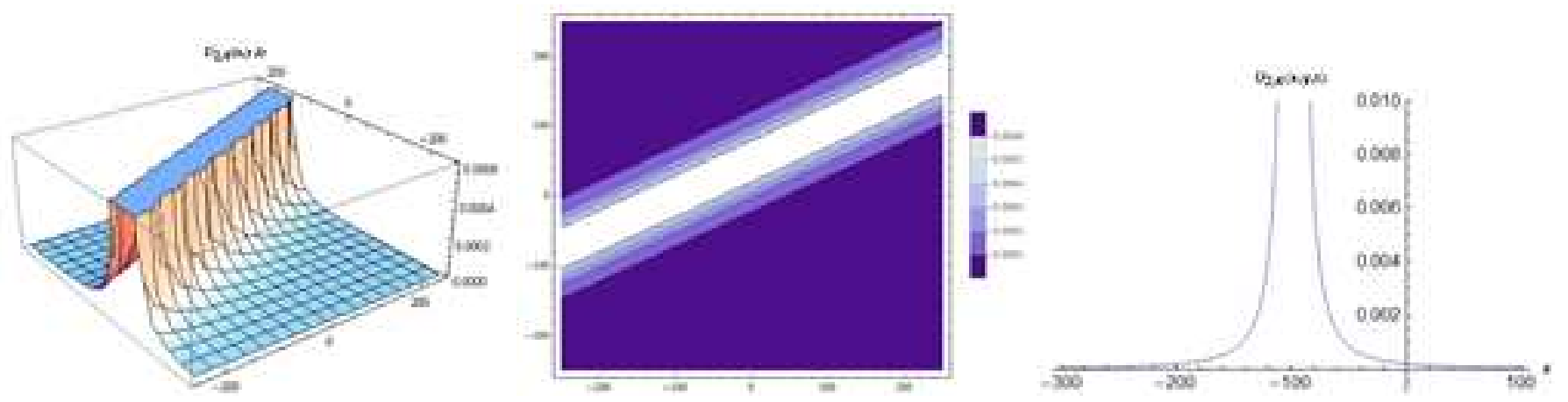

Figure 5: The 3D, contour graphic and 2D surfaces of Eq. (3.9) in $\lambda=-2, \mu=1, c=2, A_{3}=2, B_{1}=$ $1, \mathrm{EE}=100, \mathrm{t}=1$.

\section{Conclusion and Discussion}

In this study, various travelling wave solutions of the KP-BBM equation were found using the modified exponential function method. When the obtained results are analyzed, it is seen that the modified exponential function method is a suitable mathematical method for solving nonlinear partial differential equations. The two and three dimensional and contour graphics of the solutions were drawn by using the Mathematica package program. When the obtained graphs are investigated, it is seen that periodic function curves appear. Finding such functions provides the advantage that the solution can be easily interpreted in the desired range.

\section{References}

[1] T. Akturk, Y. Gurefe, H. Bulut, New function method to the $(\mathrm{n}+1)$-dimensional nonlinear problems, Int. J. Optim. Control. Theor. Appl. IJOCTA, 7 (2017), 234-239. 1

[2] T. Akturk, Y. Gurefe, H. Bulut, An application of the new function method to the Zhiber-Shabat equation, Int. J. Optim. Control. Theor. Appl. IJOCTA, 7 (2017), 271-274. 1

[3] Z. Avazzadeh, M. H. Heydari, C. Cattani, Legendre wavelets for fractional partial integro-differential viscoelastic equations with weakly singular kernels, The European Physical Journal Plus, 134 (2019), 13 pages. 1, 2

[4] H. M. Baskonus, New acoustic wave behaviors to the Davey-Stewartson equation with power-law nonlinearity arising in fluid dynamics, Nonlinear Dynam., 86 (2016), 177-183.

[5] H. M. Baskonus, New complex and hyperbolic function solutions to the generalized double combined Sinh-Cosh-Gordon equation, AIP Conf. Proc., 1798 (2017), 1-9.

[6] H. M. Baskonus, Complex soliton solutions to the Gilson-Pickering model, Axioms, 8 (2019), 14 pages. 1

[7] H. M. Baskonus, H. Bulut, On the complex structures of Kundu-Eckhaus equation via improved Bernoulli sub-equation function method, Waves Random Complex Media, 25 (2015), 720-728. 1

[8] H. M. Baskonus, H. Bulut, A. Atangana, On the complex and hyperbolic structures of the longitudinal wave equation in a magneto-electro-elastic circular rod, Smart Materials and Structures, 25 (2016), 8 pages. 1

[9] H. M. Baskonus, H. Bulut, T. A. Sulaiman, Investigation of various travelling wave solutions to the extended $(2+1)-$ dimensional quantum ZK equation, Eur. Phys. J. Plus , 132 (2017), 8 pages. 1

[10] H. M. Baskonus, H. Bulut, T. A. Sulaiman, New complex hyperbolic structures to the Lonngren-wave equation by using sine-Gordon expansion method, Appl. Math. Nonlinear Sci., 4 (2019), 141-150. 1

[11] H. M. Baskonus, T. A. Sulaiman, H. Bulut, Bright, dark optical and other solitons to the generalized higher-order NLSE in optical Fibers, Optical and Quantum Electronics, 50 (2018), 12 pages. 1

[12] J. L. Bona, On solitary waves and their role in the evolution of long waves, Applications of Nonlinear Analysis in the Physical Sciences, 1981 (1981), 183-205. 1

[13] H. Bulut, T. Akturk, Y. Gurefe, Travelling wave solutions of the ( $\mathrm{N}+1)$-dimensional sine-cosine-Gordon equation, AIP Conf. Proc., 1637 (2014), 145-149. 1

[14] H. Bulut, G. Yel, H. M. Baskonus, An application of improved Bernoulli sub-equation function method to the nonlinear time-fractional burgers equation, Turk. J. Math. Comput. Sci., 5 (2016), 1-7. 1

[15] C. Cattani, Multiscale analysis of wave propagation in composite materials, Math. Model. Anal., 8 (2003), 267-282. 1

[16] C. Cattani, Harmonic wavelet solutions of the Schrodinger equation, Int. J. Fluid Mech. Res., 30 (2003), $463-472$.

[17] C. Cattani, T. A. Sulaiman, H. M. Baskonus, H. Bulut, Solitons in an inhomogeneous Murnaghan's rod, Eur. Phys. J. Plus, 133 (2018), 11 pages. 
[18] C. Cattani, T. A. Sulaiman, H. M. Baskonus, H. Bulut, On the soliton solutions to the Nizhnik-Novikov-Veselov and the Drinfel'd-Sokolov systems, Opt. Quant. Electron., 50 (2018), 11 pages. 1

[19] Y. Chen, Z. Yan, New exact solutions of $(2+1)$-dimensional Gardner equation via the new sine-Gordon equation expansion method, Chaos Solitons Fractals, 26 (2005), 399-406. 1

[20] A. Ciancio, H. M. Baskonus, T. A. Sulaiman, H. Bulut, New structural dynamics of isolated waves via the coupled nonlinear Maccari's system with complex structure, Indian J. Phys., 92 (2018), 1281-1290. 1

[21] F. Dusunceli, New exponential and complex traveling wave solutions to the Konopelchenko-Dubrovsky model, Adv. Math. Phys., 2019 (2019), 9 pages.

[22] F. Dusunceli, Solutions for the Drinfeld-Sokolov equation using an IBSEFM method, MSU J. of Sci., 6 (2018), 505-510.

[23] F. Dusunceli, New exact solutions for the (3+1)-dimensional B-type Kadomtsev-Petviashvili equation, Erzincan University Journal of Science and Technology, 12 (2019), 463-468.

[24] S. M. El-Shaboury, M. K. Ammar, W. M. Yousef, Analytical solutions of the relative orbital motion in unperturbed and in $\mathrm{J}_{2}$-perturbed elliptic orbits, Appl. Math. Nonlinear Sci., 2 (2017), 403-414.

[25] E. I. Eskitaşçioğlu, M. B. Aktas, H. M. Baskonus, New complex and hyperbolic forms for Ablowitz-Kaup-Newell-Segur wave equation with fourth order, Appl. Math. Nonlinear Sci., 4 (2019), 105-112. 1

[26] J. H. He, X. H .Wu, Exp-function method for nonlinear wave equations, Chaos Solitons Fractals, 30 (2006), 700-708. 1, 2

[27] M. H. Heydari, M. R. Hooshmandasl, F. M. Maalek Ghaini, C. Cattani, A computational method for solving stochastic Ito-Volterra integral equations based on stochastic operational matrix for generalized hat basis functions, J. Comput. Phys., 270 (2014), 402-415. 1

[28] B. B. Kadomtsev, V. I. Petviashvili On the stability of solitary waves in weakly dispersing media, In Sov. Phys. Dokl., 15 (1970), 539-541. 1

[29] C. M. Khalique, I. E. Mhlanga, Travelling waves and conservation laws of a $(2+1)$-dimensional coupling system with Korteweg-de Vries equation, Appl. Math. Nonlinear Sci., 3 (2018), 241-253. 1

[30] N. A. Kudryashov, One method for finding exact solutions of nonlinear differential equations, Commun. Nonlinear Sci. Numer. Simul., 17 (2012), 2248-2253. 1

[31] C. S. Liu, Trial equation method and its applications to nonlinear evolution equations, Acta Phys. Sinica, 54 (2005), 2505-2509. 1

[32] S. Micu, On the controllability of the linearized Benjamin-Bona-Mahony equation, SIAM J. Control Optim., 39 (2001), 1677-1696. 1

[33] P. K. Pandey, A new computational algorithm for the solution of second order initial value problems in ordinary differential equations, Appl. Math. Nonlinear Sci., 3 (2018), 167-173. 1

[34] Y. Pandir, Y. Gurefe, U. Kadak, E. Misirli, Classification of exact solutions for some nonlinear partial differential equations with generalized evolution, Abstr. Appl. Anal., 2012 (2012), 16 pages. 1

[35] Y. Pandir, Y. Gurefe, E. Misirli, A new approach to Kudryashov's method for solving some nonlinear physical models, Int. J. Phys. Sci., 7 (2012), 2860-2866. 1

[36] D. Rani, V. Mishra, C. Cattani, Numerical inversion of Laplace transform based on Bernstein operational matrix, Math. Methods Appl. Sci., 41 (2018), 9231-9243. 1

[37] D. Rani, V. Mishra, C. Cattani, Numerical inverse Laplace transform for solving a class of fractional differential equations, Symmetry, 11 (2019), 20 pages.

[38] J. J. Rushchitsky, C. Cattani, E. V. Terletskaya, Wavelet analysis of the evolution of a solitary wave in a composite material, Int. Appl. Mech., 40 (2004), 311-318. 1

[39] G. Shen, Y. Sun, Y. Xiong, New travelling-wave solutions for Dodd-Bullough equation, J. Appl. Math., 2013 (2013), 5 pages. 1

[40] Y. Sun, New travelling wave solutions for Sine-Gordon equation, J. Appl. Math., 2014 (2014), 4 pages. 1

[41] T. A. Sulaiman, H. Bulut, A. Yokus, H. M. Baskonus, On the exact and numerical solutions to the coupled Boussinesq equation arising in ocean engineering, Indian J. Phys., 93 (2019), 647-656. 1, 2

[42] T. A. Sulaiman, A. Yokus, N. Gulluoglu, H. M. Baskonus, Regarding the Numerical and Stability Analysis of the Sharma-Tasso-Olver Equation, ITM Web Conf., 22 (2018), 9 pages. 1

[43] F. Xu, Application of Exp-function method to symmetric regularized long wave (SRLW) equation, Phys. Lett., 372 (2008), 252-257. 1

[44] X. F. Yang, Z. C. Deng, Y. Wei, A Riccati-Bernoulli sub-ODE method for nonlinear partial differential equations and its application, Adv. Difference Equ., 2015 (2015), 17 pages. 1

[45] G. Yel, H. M. Baskonus, H. Bulut, Novel archetypes of new coupled Konno-Oono equation by using sine-Gordon expansion method, Opt. Quant. Electron., 49 (2017), 10 pages. 1

[46] G. Yel, H. M. Baskonus, H. Bulut, Regarding on the some novel exponential travelling wave solutions to the Wu-Zhang system arising in nonlinear water wave model, Indian J. Phys., 93 (2019), 1031-1039.

[47] A. Yokus, Comparison of Caputo and conformable derivatives for time-fractional Korteweg-de Vries equation via the finite difference method, Internat. J. Modern Phys., 32 (2018), 12 pages.

[48] A. Yokus, S. Gulbahar, Numerical solutions with linearization techniques of the fractional Harry Dym equation, Appl. Math. Nonlinear Sci., 4 (2019), 35-41. 
[49] A. Yokus, T. A. Sulaiman, M. T. Gulluoglu, H. Bulut, Stability analysis, numerical and exact solutions of the $(1+1)$ dimensional NDMBBM equation, ITM Web Conf., 22 (2018), 10 pages.

[50] A. Yokus, M. Tuz, An application of a new version of $\left(\mathrm{G}^{\prime} / \mathrm{G}\right)$-expansion method, In AIP Conf. Proc., 1798 (2017), 7 pages. 1

[51] A. M. Wazwaz, The extended tanh method for new compact and noncompact solutions for the KP-BBM and the ZK-BBM equations, Chaos Solitons Fractals, 38 (2008), 1505-1516. 1 